ORIGINAL ARTICLE

\title{
Increasing incidence of metastatic prostate cancer in the United States (2004-2013)
}

\author{
AB Weiner ${ }^{1}$, RS Matulewicz ${ }^{1}$, SE Eggener ${ }^{2}$ and EM Schaeffer ${ }^{1}$
}

BACKGROUND: Changes in prostate cancer screening practices in the United States have led to recent declines in overall incidence, but it is unknown whether relaxed screening has led to changes in the incidence of advanced and metastatic prostate cancer at diagnosis.

METHODS: We identified all men diagnosed with prostate cancer in the National Cancer Data Base (2004-2013) at 1089 different health-care facilities in the United States. Joinpoint regressions were used to model annual percentage changes (APCs) in the incidence of prostate cancer based on stage relative to that of 2004.

RESULTS: The annual incidence of metastatic prostate cancer increased from 2007 to 2013 (Joinpoint regression: APC: 7.1\%, $P<0.05)$ and in 2013 was $72 \%$ more than that of 2004 . The incidence of low-risk prostate cancer decreased from years 2007 to 2013 (APC: $-9.3 \%, P<0.05$ ) to $37 \%$ less than that of 2004 . The greatest increase in metastatic prostate cancer was seen in men aged 55-69 years (92\% increase from 2004 to 2013).

CONCLUSIONS: Beginning in 2007, the incidence of metastatic prostate cancer has increased especially among men in the age group thought most likely to benefit from definitive treatment for prostate cancer. These data highlight the continued need for nationwide refinements in prostate cancer screening and treatment.

Prostate Cancer and Prostatic Diseases (2016) 19, 395-397; doi:10.1038/pcan.2016.30; published online 19 July 2016

\section{INTRODUCTION}

Over the past decade, there has been substantial shifts in PSA screening practices in response to randomized trial data and US Preventive Services Task Force (USPSTF) recommendations in 2008 and 2012. ${ }^{1,2}$ This has resulted in reduced screening with associated declines in overall prostate cancer incidence. ${ }^{3,4}$ Using data from a large cancer registry from 2004 through 2013, we sought to analyze the temporal relationship between relaxed screening and increases in yearly incidence of advanced or metastatic prostate cancer.

\section{MATERIALS AND METHODS}

From the National Cancer Data Base (NCDB), all men diagnosed with adenocarcinoma of the prostate (International Classification of Diseases for Oncology histology codes 8550 and 8140) from 2004 through 2013 were included. Only patients with data available to risk stratify based on National Comprehensive Cancer Network (NCCN) guidelines were included (low risk: CT1 cT2a, PSA $<10 \mathrm{ng} \mathrm{ml}^{-1}$ and Gleason score $\leqslant 6$; intermediate risk: cT2b-T2c, PSA $10-20 \mathrm{ng} \mathrm{ml}^{-1}$ and Gleason score 7; high risk: cT3-4, PSA $\geqslant 20 \mathrm{ng} \mathrm{ml}^{-1}$ and Gleason score 8-10; metastatic $\mathrm{CN} 1$ or $\left.\mathrm{CM} 1\right) .^{5}$ To avoid bias in temporal trends, the analysis was limited to facilities that contributed prostate cancer patient data each year during the study period. After those facilities were excluded, a total of 767550 patients from 1089 facilities were analyzed.

The primary outcome was annual incidence of prostate cancer based on NCCN risk groups relative to that of 2004. That is, the outcome denominator was the incidence in 2004 and the numerator was the incidence for every year after. Patients were considered to have metastatic prostate cancer if they were found to have clinically nodal or distant metastases ( $\mathrm{cN} 1$ or $\mathrm{CM} 1)$ at the time of diagnosis. To model temporal trends in incidence, Joinpoint software 4.2.0.2 (http://surveillance.cancer. gov/joinpoint/) was used to estimate annual percent changes (APCs) in incidence relative to 2004 and to identify significant changes in the linear slope of annual prostate cancer incidence stratified by individual risk and age groups. A two-sided $P<0.05$ was used to determine whether APCs were statistically significant. The Mann-Whitney U-test was used to compare annual median diagnostic PSA values among men with metastatic prostate cancer. This study of de-identified, publicly available data was determined to be exempt by the Northwestern University Institutional Review Board.

\section{RESULTS}

Of the 767550 men with prostate cancer included in our study, $3 \%$ had metastases at diagnosis. The remainder had localized disease: $32 \%$ low, $45 \%$ intermediate and $20 \%$ high risk (Table 1). ${ }^{5}$ Relative to 2004, the annual incidence of all prostate cancer did not change significantly over time (Figure 1 and Table 1). The incidence of low-risk prostate cancer decreased from years 2007 to 2013 (Joinpoint regression, APC: $-9.3 \%, P<0.05$ ) to $37 \%$ less than that of 2004 (25 708 cases in 2004 to 16223 in 2013). Intermediate-risk prostate cancer increased from 2004 to 2008 (APC: $10.0 \%, P<0.05)$ and did not change significantly in the following years (APC $-2.7 \%, P=0.3$ ). The incidence of intermediate-risk prostate cancer was 23\% more in 2013 compared with 2004. Similarly, the incidence of high-risk prostate cancer rose from 2004 to 2008 and declined thereafter, although these changes were not statistically significant. Conversely, the incidence of metastatic prostate cancer rose from 2007 to 2013 (APC: 7.1\%, $P<0.05$ ) increasing to $72 \%$ more than that of 2004 (1685 cases in 2004 to 2890 in 2013).

\footnotetext{
${ }^{1}$ Department of Urology, Northwestern University Feinberg School of Medicine, Chicago, IL, USA and ${ }^{2}$ Section of Urology, Department of Surgery, University of Chicago, Chicago, IL, USA. Correspondence: Dr EM Schaeffer, Department of Urology, Northwestern University Feinberg School of Medicine, 393 East Chicago Avenue, Tarry Building 16-713, Chicago, IL 60611, USA.

E-mail: e-schaeffer@northwestern.edu

Received 21 June 2016; revised 28 June 2016; accepted 30 June 2016; published online 19 July 2016
} 
Table 1. Annual incidence of prostate cancer based on National Comprehensive Cancer Network risk group in the National Cancer Data Base

Year of Low risk Intermediate risk High risk Metastatic All diagnosis

\begin{tabular}{rrrrrr}
\hline 2004 & 25708 & 27347 & 14074 & 1685 & 68814 \\
2005 & 25993 & 28761 & 14121 & 1947 & 70822 \\
2006 & 28509 & 32753 & 15126 & 1909 & 78297 \\
2007 & 30323 & 36766 & 16256 & 1884 & 85229 \\
2008 & 29327 & 40201 & 16870 & 2062 & 88460 \\
2009 & 23643 & 34497 & 14627 & 2135 & 74902 \\
2010 & 24264 & 38628 & 15630 & 2584 & 81106 \\
2011 & 24626 & 40394 & 15822 & 2609 & 83451 \\
2012 & 18284 & 34215 & 14203 & 2697 & 69399 \\
2013 & 16223 & 33578 & 14379 & 2890 & 67070 \\
Total & 246900 & 347140 & 151108 & 22402 & 767550
\end{tabular}

Annual data are reported from 1089 facilities that contributed prostate cancer patient data during each year of the study period.

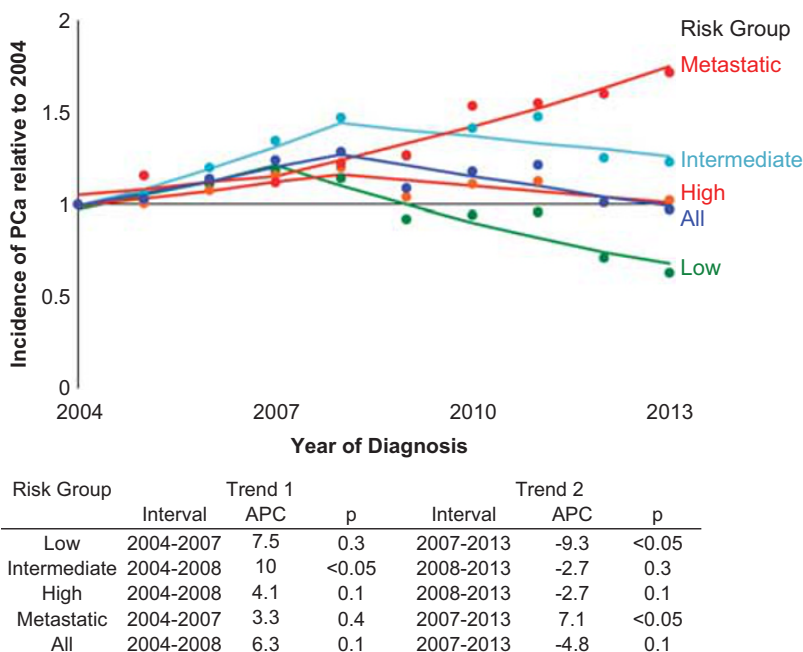

Figure 1. Annual incidence of prostate cancer based on the NCCN risk group relative to 2004 in the United States. Joinpoint regressions were used to model linear trends and determine statistical significance. Trend 1 represents an initial best fit line, whereas trend 2 represents a second linear fit if there is a change in trend from the initial line. The incidence of metastatic prostate cancer has increased recently by $72 \%$, whereas the incidence of low-risk prostate cancer decreased by $37 \%$. APC, annual percentage change; NCCN, National Comprehensive Cancer Network; PCa, prostate cancer.

The increasing incidence of metastatic prostate cancer was statistically significantly in all age groups with the largest increase seen in men aged 55 to 69 years from 702 cases in 2004 to 1345 in 2013 (92\% increase; Figure 2 and Table 2). Whereas median PSA levels among men diagnosed with localized disease were stable over the study period $\left(6.1 \mathrm{ng} \mathrm{ml}^{-1}\right.$ (interquartile range (IQR) $4.5-9.6 \mathrm{ng} \mathrm{ml}^{-1}$ ) to $\left.6.2 \mathrm{ng} \mathrm{ml}^{-1}\left(\mathrm{IQR} 4.6-9.6 \mathrm{ng} \mathrm{ml}^{-1}\right)\right)$, the median PSA levels among men with metastatic prostate cancer increased from $25.5 \mathrm{ng} \mathrm{ml}^{-1}$ (IQR 10.4-67.5 $\mathrm{ng} \mathrm{ml}^{-1}$ ) in 2004 to $49.7 \mathrm{ng} \mathrm{ml}^{-1}$ (IQR 14.3-98.0 $\left.\mathrm{ng} \mathrm{ml}^{-1}\right)$ in $2013(P<0.001)$.

\section{DISCUSSION}

Since 2007, the incidence of metastatic prostate cancer has risen significantly whereas that of low-risk prostate cancer has simultaneously fallen. The largest increase in metastatic prostate

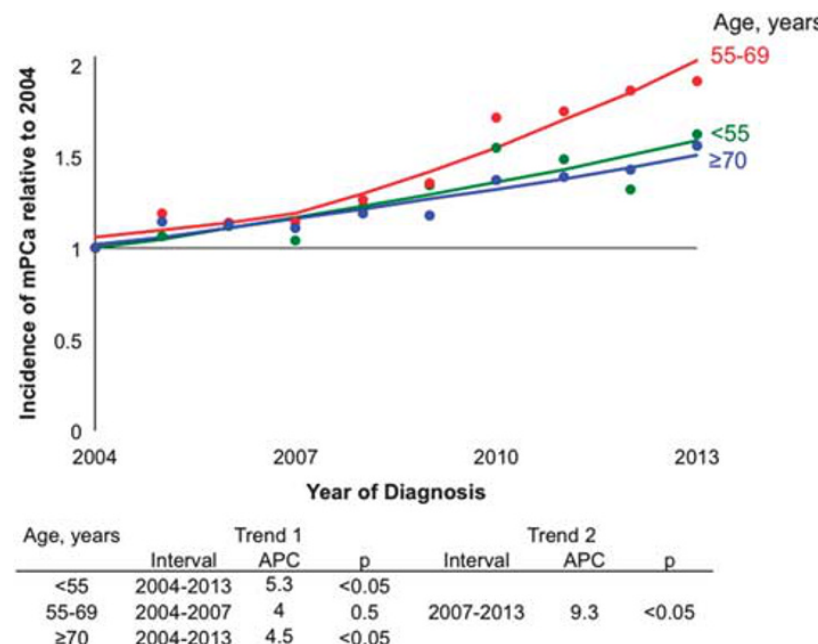

Figure 2. Annual incidence of metastatic prostate cancer based on age relative to 2004 in the United States. Joinpoint regressions were used to model linear trends and determine statistical significance. Trend 1 represents an initial best fit line, whereas trend 2 represents a second linear fit if there is a change in trend from the initial line. The incidence of metastatic prostate cancer has increased recently in all age groups, with the largest increases seen in men aged 55-69 $(92 \%)$ years. APC, annual percentage change; $\mathrm{mPCa}$, metastatic prostate cancer.

Table 2. Annual incidence of metastatic prostate cancer based on age in the National Cancer Data Base

\begin{tabular}{lrrrr}
\hline Year of diagnosis & $<55$ & $55-69$ & $\geqslant 70$ & \multicolumn{1}{c}{ All } \\
\hline 2004 & 166 & 702 & 817 & 1685 \\
2005 & 177 & 836 & 934 & 1947 \\
2006 & 186 & 800 & 923 & 1909 \\
2007 & 173 & 804 & 907 & 1884 \\
2008 & 203 & 888 & 971 & 2062 \\
2009 & 223 & 951 & 961 & 2135 \\
2010 & 257 & 1205 & 1122 & 2584 \\
2011 & 247 & 1229 & 1133 & 2609 \\
2012 & 219 & 1308 & 1170 & 2697 \\
2013 & 269 & 1345 & 1276 & 2890 \\
Total & 2120 & 10068 & 10214 & 22402 \\
\hline
\end{tabular}

Annual data are reported from 1089 facilities that contributed prostate cancer patient data during each year of the study period.

cancer was seen among the age group (55-69 years) thought to benefit most from prostate cancer screening and early definitive treatment. ${ }^{6}$ These findings cannot be explained completely by reactions to the USPSTF recommendations alone, as increases in metastatic prostate cancer began in the years before its release. Changes in aggregate screening before the USPSTF grade D recommendation, alterations in the biological aggressiveness of prostate cancer or increases in the ascertainment of metastatic disease (through increased sensitivity and utilization of imaging) are plausible explanations. Although the latter is possible, it seems less likely given increased imaging would discover metastases in more men with lower PSAs and we noted the opposite, a simultaneous increase in PSAs among men with metastatic prostate cancer.

Although the social and biologic factors underlying these PSA escapes and rising metastatic prostate cancer cases are unknown, the implications of these recent trends highlight the continued 
need for nationwide refinements in prostate cancer screening and treatment to prevent the morbidity and mortality associated with metastatic prostate cancer. This will be particularly critical for population health economics in the United States considering the added cost of care for metastatic prostate cancer ${ }^{7}$ and an aging constituency whose population over the age 65 years will double to over a projected 80 million by the year $2050{ }^{8}$ In addition, our findings and forthcoming changes in the number of elderly individuals should provide impetus to improve treatments for men with metastatic prostate cancer whose cancer-specific survival has not changed significantly in the past two decades. ${ }^{9}$

Data from the NCDB also demonstrated an increase in the incidence of intermediate-risk prostate cancer in the early years of the study period. This likely reflects grade migration because of modifications in the Gleason Grading system in 2005 by the International Society of Urologic Pathology leading to Gleason 6 cancers being upgraded. ${ }^{10}$ Declines seen in low-risk prostate cancer incidence following the year 2007 likely reflects changes in aggregate screening after the 2008 USPSTF recommendation against screening for the elderly. ${ }^{1}$

Limitations to the current study include the lack of national annual incidence rates in the NCDB. Thus, our outcome variable was annual incidence of prostate cancer at over 1000 health-care facilities in the United States relative to that of 2004, the initial year of our study period. However, given the large number of patients and health-care facilities analyzed, it is likely that trends in the NCDB reflect national patterns.

\section{CONFLICT OF INTEREST}

The authors declare no conflict of interest.

\section{ACKNOWLEDGEMENTS}

This paper was supported by grants NIH 5U01CA196390 and the Prostate Cancer Foundation - Hagen Special Challenge.

\section{REFERENCES}

1 U. S. Preventive Services Task Force. Screening for prostate cancer: U.S. Preventive Services Task Force recommendation statement. Ann Intern Med 2008; 149: 185-191.

2 Moyer VAU. S. Preventive Services Task Force. Screening for prostate cancer: U.S. Preventive Services Task Force recommendation statement. Ann Intern Med 2012; 157: $120-134$

3 Jemal A, Fedewa SA, Ma J, Siegel R, Lin CC, Brawley O et al. Prostate cancer incidence and PSA testing patterns in relation to USPSTF screening recommendations. JAMA 2015; 314: 2054-2061.

4 Drazer MW, Huo D, Eggener SE. National prostate cancer screening rates after the 2012 US Preventive Services Task Force recommendation discouraging prostatespecific antigen-based screening. J Clin Oncol 2015; 33: 2416-2423.

5 Mohler JL, Armstrong AJ, Bahnson RR, D'Amico AV, Davis BJ, Eastham JA et al. Prostate cancer, version 1.2016. J Natl Compr Canc Netw 2016; 14: 19-30.

6 Carter HB, Albertsen PC, Barry MJ, Etzioni R, Freedland SJ, Greene KL et al. Early detection of prostate cancer: AUA guideline. J Urol 2013; 190: 419-426.

7 Yabroff KR, Lamont EB, Mariotto A, Warren JL, Topor M, Meekins A et al. Cost of care for elderly cancer patients in the United States. J Natl Cancer Inst 2008; 100: 630-641.

8 Ortman JM, Velkoff VA, Hogan HAn Aging Nation: The Older Population in the United StatesCurrent Population Reports, P25-1140. US Census Bureau: Washington, DC, 2014

9 Wu JN, Fish KM, Evans CP, Devere White RW, Dall'Era MA. No improvement noted in overall or cause-specific survival for men presenting with metastatic prostate cancer over a 20-year period. Cancer 2014; 120: 818-823.

10 Weiner AB, Etzioni R, Eggener SE. Ongoing Gleason grade migration in localized prostate cancer and implications for use of active surveillance. Eur Urol 2014; 66 : 611-612. 